\title{
Crack Identification of Drawing Parts Based on Local Wave Demomposition and Neural Network
}

\author{
Zhigao Luo, Qiang Chen \\ Department of Mechnical Engineering \\ Jiangsu University \\ Zhenjiang, Jiangsu Province, China \\ lzg3359@163.com
}

\author{
Xin He, Aicheng Xu \\ Department of Mechnical Engineering \\ JiangSu University \\ Zhenjiang, Jiangsu Province, China \\ star-ren@126.com
}

\begin{abstract}
This paper relates to local wave decomposition and back-propagation (BP) neural network.With local wave method, an arbitrary acoustic emission signal can be decomposed efficiently and accurately into a set of intrinsic mode functions (IMFs) and a residual trend. The energy feature parameters extracted from IMFs were employed as the input parameters of the neural network to identify the acoustic emission signals of drawing parts. The experimental results showed this method was effective for crack identification of drawing parts.
\end{abstract}

Keywords- Acoustic emission; Local wave; Back-propagation neural network; Drawing parts; Crack

\section{INTRODUCTION}

Because the working conditions of the mold is extremely severe, drawing parts that withstand high contact pressure and severe friction in forming process will inevitably come into being cracks. Some macroscopic cracks are imperceptible, the bulk of the waste defective has produced before discovering, which bring huge economic losses to enterprise. Obviously, how to realize the crack online monitoring of the metal drawing parts.

Crack identification is a subject of great technical problem. The foremost problem is how to eliminate noise and other signals to extract accurately acoustic emission signal feature of the crack, it is the bottleneck problem for current crack identification, which relates directly to the accuracy of the fault diagnosis and the reliability of the early stage prediction. In this paper, the method of timefrequency analysis, local wave decomposition and the pattern recognition technique, back-propagation (BP) neural network, are combined and applied to crack identification of drawing parts.

\section{LOCAL WAVE DECOMPOSITION}

Local wave theory is brought out based on the illumination of the empirical mode decomposition(EMD) method by American scholars Huang. In 1996, an American scholar Huang proposed firstly a mode decomposition method based on the experience in an international conference which is suitable for analyzing the nonstationary signal. In 2000, through analyzing thoroughly the non-stationary signal inherent characteristics, Ma Xiaojiang professor is the first to present theconcept of local wave, combing Huang's thoughts and EMD algorithm.

Local wave decomposition aims at that a complex nonlinear and nonstationary signal is decomposted into a sum of intrinsic mode functions (IMFs ) from the most to the least frequent,according to local time scale characteristics. An IMF is a function that satisfies two conditions:Firstly, the number of local extrema and the number of zero crossings must be equal or differ by 1 at most; Secondly at any point the mean value of the envelope defined by the local maxima and the envelope defined by the local minima must be zero.

A collected acoustic emission signal is decomposted into $n$ IMFs and a trend component,as shown in (1).

$$
x(t)=\sum_{i=1} c_{i}(t)+r_{n} \quad(i=1,2, \ldots, n)
$$

Symbols Regarding an arbitrary data series $\mathrm{x}(\mathrm{t})$, the IMFs are obtained,using the following algorithm, shown by Schlurmann.

1) Initialize: $r_{0}(t)=x(t), i=1$

2) Extract the $i$ th IMF:

(a) Initialize: $h_{0}(t)=r_{i}(t), k=1$.

(b) Extract the local maxima and minima of $h_{k-1}(t)$.

(c) Interpolate the local maxima and the local minima by a cubic spline to form upper and lower envelopes of $h_{k-1}(t)$.

(d) Calculate the mean $m_{k-1}(t)$ of the upper and lower envelopes of $h_{k-1}(t)$.

(e) Define: $h_{k}(t)=h_{k-1}(t)-m_{k-1}(t)$.

(f) If IMF criteria are satisfied, then set $\operatorname{IMF}_{i}(t)=h_{k}(t)$ else go to (b) with $k=k+1$.

3) Define: $r_{i}(t)=r_{i-1}(t)-\operatorname{IMF}_{i}(t)$.

4) If $r_{i}(t)$ still has at least two extrema, then go to 2$)$ with $i=i+1$; else the decomposition is completed and $r_{i}(t)$ is the "residue" of $x(t)$. 


\section{EXTRACTION OF THE ENERGY FEATURE PARAMETERS FROM IMFS}

After the local wave decomposition,the local feature can be highlighted. The crack feature can be accquised more accurately and effectively through analyzing these IMFs. When acoustic emission signals generate the energy of each frequency band will change. These energy contains abundant information, so the energy of each IMF can be extracted to analyze the feature of crack. The steps can be summarized as following:

1) Decompose an arbitrary acoustic emission signal into a set of intrinsic mode functions (IMFs) and a residual trend, as shown in (1).

2) Find the energy $e_{i}$ of each of IMFs, as shown in (2).

$$
e_{i}=\int_{-\infty}^{\infty}\left|c_{i}(t)^{2}\right| d t \quad(i=1,2, \ldots, n)
$$

3) Construct the characteristic vector $X$ consisting of energy elements as shown in (3).

$$
X=\left[e_{1}, e_{2}, \ldots, e_{n}\right] \quad(i=1,2, \ldots, n)
$$

When the energy is a larger value, $e_{i}$ typically is also a larger number, this isn't inconvenient for data analysis. Thus, the vector $X$ should be normalized .After normalization, the characteristic vector $T$ is obtained,as shown in (4) and (5).

$$
\begin{gathered}
e=\left(\sum_{i=1}^{n}\left|e_{i}\right|^{2}\right)^{\frac{1}{2}} \quad(i=1,2, \ldots, n) \\
T=\left[e_{1} / e, e_{2} / e, \ldots \ldots, e_{n} / e\right] \quad(i=1,2, \ldots, n) .
\end{gathered}
$$

\section{FUNDAMENTAL PRINCIPLES OF BACK- PROPAGATION (BP) NEURAL NETWORKS}

BP neural network is a neural network of most extensive application and most mature development. it is a multilayer forward networks with the back propagation learning algorithm applied, it make up of the input layer, the output layer and the hidden layer. the nodes between layer and layer connect with a topological structure of connection and it have good self-learning and classification ability. it can be used to identify the type of fault in fault diagnosis.

The working process of BP network is divided into two stages. The first stage is positive transmission process and the second stage is the reverse error modified process. The value for a given network is transported to the hidden layer with the process of weighting. The value handled by the hidden stimulate function is the input of output layer. Then the value handled by the stimulate function in output layer is the final output. The process is a positive update step by step. If the error exist between the output value and a given expectations and not satisfy the requirement of accuracy, it will go into error inverse propagation process. it will make the error go into the step back propagation and correct the value in each layer. It will repeat the above two stages until the error meets the demand of accuracy. the train will stop. The whole process is usually said to be the BP algorithm.
In this paper, the energy feature parameters extracted from IMFs were employed as the input parameters of BP neural network to identify the acoustic emission (AE) signals of drawing parts.

\section{EXAMPLE OF DIAGNOSIS}

\section{A. Signal Acquisition}

Define The test was completed in the intelligent 350T inhydraulic compressor of Zhenjiang Sanwei Conveying Eqiupment Co.,Ltd . Extrusion part was bucket, its dimension

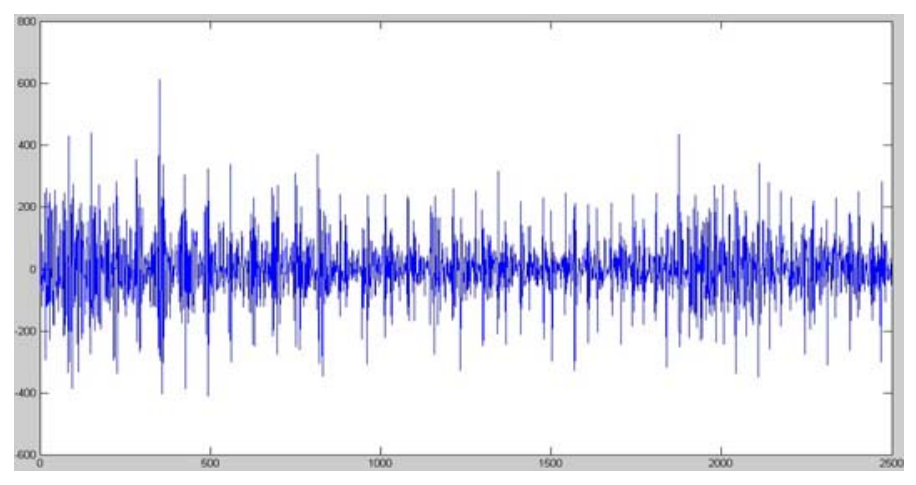

Fig. 1 AE signal $x_{1}$ from normal state of drawing parts.

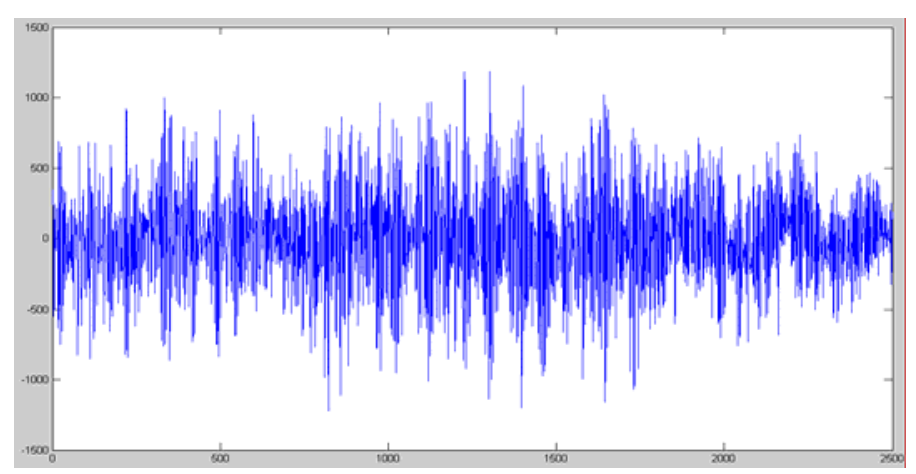

Fig. 2 AE signal $x_{2}$ from crack state of drawing parts.

is $20 \mathrm{~mm} * 200 \mathrm{~mm}$.PengXiang Waveform Acoustic Emission Instument of Beijing Pengxiang Technology Co..Ltd was employed to collect acoustic emission signals from extrusion parts. It concludes PXR15 AE sensors(Resonant Frequency is150 kHz),Pre-Amplfier(Gain is 40dB),Data Acquisition Card(AE input channels are four and A/D sampling rate is $20 \mathrm{MSps}$ ).

Fifteen normal signals and fifteen crack signals were collected by PengXiang Waveform Acoustic Emission Instument, Fig. 1 shows a original acoustic emission signal collected from normal state of drawing parts,and Fig.2 shows a original acoustic emission signal collected from crack state of drawing parts,set them $x_{1}, x_{2}$. 


\section{B. Extraction of Characteristic Parameters}

Firstly each of thirty groups collected AE signals was decomposted into a set of intrinsic mode functions (IMFs ) by using the method of local wave decomposition ,Fig.3 shows the IMFs of the norml AE signal $x_{1}$ after the local wave decomposition, ten IMFs and a residual trend were got; Fig.4 shows the IMFs of the crack AE signal $x_{2}$ after the local wave decomposition, eight IMFs and a residual trend were gotConsidering that feature information mainly gathered in high frequency band,the first 8 IMFs were collected to calculate their energy ,then normalized, and then characteristic parameter $T$ was got, as shown in (6).

$$
T=\left[e_{1} / e, e_{2} / e, e_{3} / e, e_{4} / e, e_{5} / e, e_{6} / e, e_{7} / e, e_{8} / e\right] .
$$

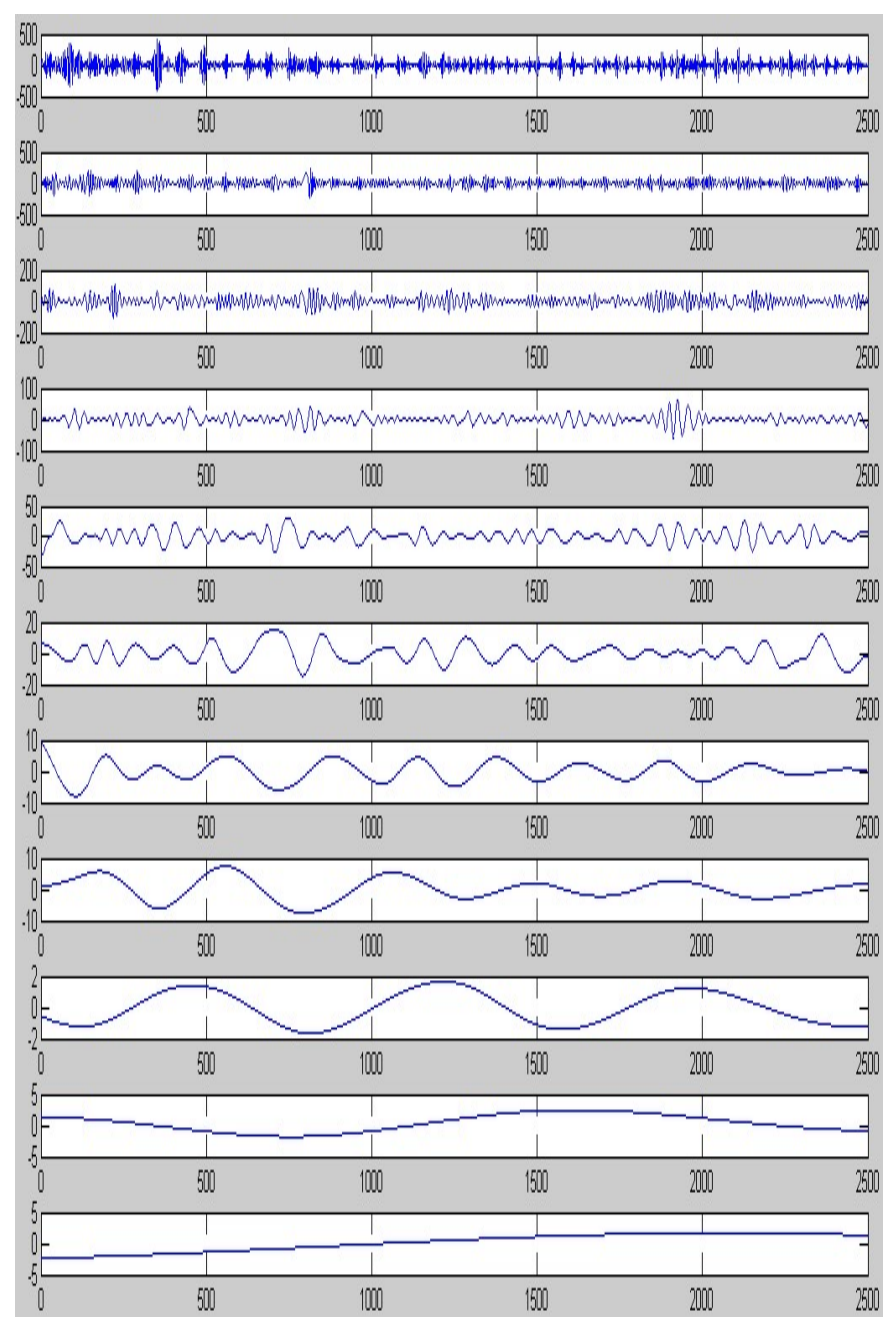

Fig. 3 Local wave decomposition of AE signal $x_{1}$.

\section{Pattern Recognition}

Design a back-propagation (BP) neural network, it was made up of the input layer, the hidden layer and the output layer .There are 8 nodes corresponding to the characteristic vector $\mathrm{T}$ after separating from the local wave in the Input layer. By the test and comparison, the nodes of the hidden layer are eleven. The output layer has two nodes corresponding to normal state and crack state. Each cases have 10 learning samples, Learning samples for the two conditions. The learning samples are trained and the output is 000 and 111 , corresponding to normal state and crack state of drawing parts. The rest five normal signal samples and five crack signal samples were input the BP neural network. TABLE I shows the diagnosis results of the BP neural network.Finally, classification models of backpropagation (BP) neural network accord with actual models of test samples. The ten test samples were all identified successfully by back-propagation (BP) neural network.

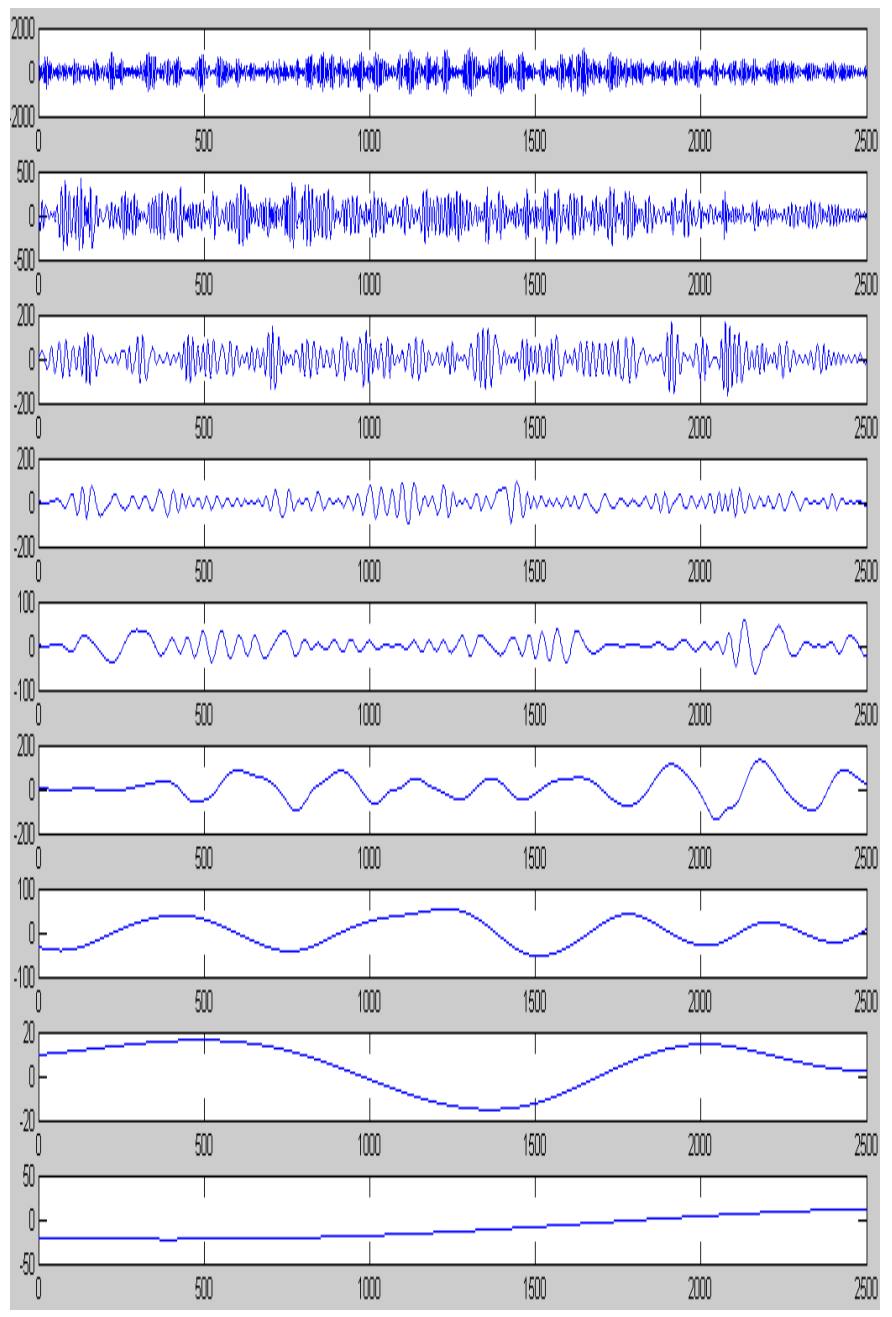

Fig. 4 Local wave decomposition of AE signal $X_{2}$. 
TABLE I The Diagnosis Results of BP Neural Network

\begin{tabular}{|c|c|c|c|}
\hline \multirow{2}{*}{ Input } & \multicolumn{2}{|c|}{ Output } & \multirow{2}{*}{ Types } \\
\cline { 2 - 3 } & Expected Values & Actual Values & \\
\hline Test1 & 000 & 0.02370 .01110 .0105 & Normal \\
\cline { 2 - 3 } Test2 & 000 & 0.00040 .00130 .0005 & Normal \\
\cline { 2 - 3 } Test3 & 000 & 0.00050 .00150 .0006 & Normal \\
\cline { 2 - 4 } Test4 & 000 & 0.05010 .05540 .0495 & Normal \\
\cline { 2 - 4 } Test5 & 000 & 0.01970 .01110 .0137 & Normal \\
\cline { 2 - 4 } Test6 & 111 & 0.94740 .97620 .9536 & Crack \\
\cline { 2 - 4 } Test7 & 111 & 0.93250 .97100 .9407 & Crack \\
\cline { 2 - 4 } Test8 & 111 & 0.98750 .99660 .9899 & Crack \\
\cline { 2 - 4 } Test9 & 111 & 0.92350 .95210 .9473 & Crack \\
\cline { 2 - 4 } Test10 & 111 & 0.91460 .93210 .9273 & Crack \\
\hline
\end{tabular}

\section{CONCLUSIONS}

Acoustic emission signal generated from crack of extrusion part is a kind of typical nonlinear and nonstationary random signal. It is trapped hardly and its characteristic parameters are extacted with difficulty. Each of IMFs contain and highlight different local feature information. Back-propagation (BP) neural network has a strong nonlinear mapping performance and a flexible network structure, so it has a excellent pattern classification ability. The method combining local wave decomposition with back-propagation (BP) neural network can effectively identify the acoustic emission signals from cracks of drawing parts.

\section{ACKNOWLEDGMENT}

The authors want to thank my graduate students Ye Hongying Chen Baogang, and Fan Xiangwei for their help and support in making this work as possible as they can. Special thanks go to Chen Baolei for helpful discussions.

\section{REFERENCES}

[1] Wang Hongyu .Synthesis and Unification Method inCorrelation Theory of Signal Processing.Beijing:National Defense Industry Press,2005.

[2] Ma Xiaojiang,Yu Bo,Zhang Zhixin.A New Approach to TimeFrequency Analysis-Local Wave Method. College Journal of Vibration Engineering, 2000, 13 (9) : 24一29.

[3] Fecit Scientific Products R\&D Center. Neural Network Theory and Matlab 7 Application.Beijing:Pubishing House of Electronics Indusry,2005.

[4] Huang $\mathrm{N}$ E, Shen Z, Long $\mathrm{S}$ R et al. The empirical mode decomposition and Hilbert spectrum for nonlinear and non-stationary time series analysis. Proc. Roy. Soc.

[5] Schlurmann, T., 2002. Spectral frequency analysis of nonlinear water waves derived from the Hilbert-Huang transformation. Journal of Offshore Mechanics and Arctic Engineering (JOMAE), American Society of Mechanical Engineers (ASME) 124 (1), 22-27.

[6] London A,1998, 454: 903-995.G. Rilling, P. Flandrin, P. Goncalves, On empirical mode decomposition and its algorithms, IEEE-EURASIP Workshop on Nonlinear Signal and Image Processing, NSIP-03, 2003, pp. 1-5.

[7] Yu, Dejie Cheng, Junsheng, Yang, Yu. Application of EMD method and Hilbert spectrum to the fault diagnosis of roller bearings. Mechanical Systems and Signal Processing, 2005, 19( 2): 259-270 